\title{
Answer to Medical Quiz: Images
}

\section{Acute transverse myelitis (TM)}

\section{Answer key}

1. MRI of cervical spine with contrast showing spinal cord swelling and hyper intense signal change in cervical spine cord.

2. longitudinally extensive transverse myelitis

3. Spinal multiple sclerosis

4. IV. Pulse methylprednisolone.

\section{Review}

Acute transverse myelitis (TM) is a rare acquired neuroimmune spinal cord disorder that can present with the rapid onset of weakness, sensory alterations, and bowel or bladder dysfunction. TM can occur as an independent entity, usually as a postinfectious complication, but TM also exists on a continuum of neuro-inflammatory disorders that includes acute disseminated encephalomyelitis, multiple sclerosis, and neuromyelitis optica. ${ }^{1}$

TM can occur as part of the spectrum of multiple sclerosis. In some cases, TM is the initial demyelinating event that proceeds clinically definite multiple sclerosis. TM manifesting as a longitudinally extensive spinal cord lesion spanning three or more vertebral segments is one of the characteristic manifestations, along with bilateral optic neuritis, of neuromyelitis optica. However, neuromyelitis optica can also cause TM involving fewer segments. ${ }^{2}$
It is important in the evaluation of patients with acute myelopathies to exclude compressive and noninflammatory causes of myelopathy as well as to distinguish various types of TM, since the prognosis, risk of recurrence, and treatment options may differ among these distinct entities. ${ }^{3}$

Intravenous glucocorticoids have long been considered the standard of care and first-line therapy in acute idiopathic TM. Even without placebo-controlled trials evaluating glucocorticoids specifically in TM there is good evidence that intravenous glucocorticoids are effective in acute inflammatory central nervous system diseases like TM, such as multiple sclerosis. ${ }^{4}$

\section{References}

1. Kaplin AI, Krishnan C, Deshpande DM, et al. Diagnosis and management of acute myelopathies. Neurologist 2005; 11:2.

2. Christensen PB, Wermuth L, Hinge HH, Bømers K. Clinical course and long-term prognosis of acute transverse myelopathy. Acta Neurol Scand 1990; 81:431.

3. Krishnan C, Kaplin AI, Pardo CA, et al. Demyelinating disorders: update on transverse myelitis. Curr Neurol Neurosci Rep 2006; 6:236.

4. Scott TF, Frohman EM, De Seze J, et al. Evidence-based guideline: clinical evaluation and treatment of transverse myelitis: report of the Therapeutics and Technology Assessment Subcommittee of the American Academy of Neurology. Neurology 2011; 77:2128. 